\title{
Nonlinear analysis of the forced response of structural elements
}

\author{
Ali H. Nayfeh, Dean T. Mook, and Seshadri Sridhar \\ Department of Engineering Science and Mechanics, Virginia Polytechnic Institute and State University, \\ Blacksburg, Virginia 24061 \\ (Received 5 July 1973)
}

\begin{abstract}
A general procedure is presented for the nonlinear analysis of the forced response of structural elements to harmonic excitations. Internal resonances (i.e., modal interactions) are taken into account. All excitations are considered, with special consideration given to resonant excitations. The general procedure is applied to clamped-hinged beams. The results reveal that exciting a higher mode may lead to a larger response in a lower interacting mode, contrary to the results of linear analyses.
\end{abstract}

Subject Classification: $40.30,40.22$.

\section{INTRODUCTION}

Slender beams and thin plates supported in such a way as to restrict movement at the ends and along the edges experience mid-plane stretching when deflected. The influence of this stretching on the dynamic response increases with the amplitude of the response. Consequent$1 y$; an analysis which accounts for mid-plane stretching can provide insight into the relatively large-amplitude oscillations that might exist in a resonant situation.

Woinowsky-Krieger ${ }^{1}$ and Burgreen ${ }^{2}$ considered the free oscillations of a beam having hinged ends a fixed distance apart. For slender beams this situation can be accurately described with nonlinear strain-displacement equations and a linear stress-strain law. Expressing the solution as a product of a function of time and a linear free-oscillation mode, they solved the nonlinear equation for the temporal function exactly in terms of Jacobi-elliptic functions. Burgreen also considered vibrations about the buckled configuration and conducted an experiment, the results of which basically supported the conclusions of the analysis. In these studies (including Burgreen's experiment), no consideration was given to the possibility of modal coupling or interaction, in spite of the fact that it seems unlikely that the deflection curve for large amplitudes is of the same form as the one for small amplitudes.

MacDonald ${ }^{5}$ worked with the same governing equations but did not consider axial prestressing, as Burgreen did. He improved the analysis by letting the deflection curve be represented at any instant by a Fourier expansion in terms of the eigenfunctions of the linear problem; i.e., the linear, free-oscillation modes. If the coefficients are functions of time, the expansion can continuously represent the deflection curve. MacDonald was able to solve the nonlinear equations for the coefficients in terms of elliptic functions. He commented that the problem is inherently nonlinear even for small-amplitude vibrations and there is always dynamic coupling of the modes. In spite of this, there followed a number of papers on nonlinear vibrations of beams and plates which did not consider the modal interactions. Some authors did, however, note the possibility. (Some additional references are included.)
Following MacDonald's approach, Bennett and Eisley ${ }^{6}$ also represented the deflection in terms of the linear modes and considered the forced oscillations of a beam having clamped ends. But apparently they did not consider the possibility of a harmonic excitation near one natural frequency exciting other natural frequencies. The results of their analysis and experiment indicate that the response is dominated by the first mode when the excitation is near the first natural frequency, but they also found higher harmonics. In a later paper, Bennett ${ }^{7}$ studied the ultraharmonic motion of a beam with hinged ends. The analyses were based on the method of harmonic balance. In both papers the stability of the solutions was studied.

Following a similar approach. Tseng and Dugundji ${ }^{8}$ represented the deflection curve with an expansion in terms of the linear, static buckling modes in studying the forced vibrations of a buckled beam. They did not find modal interactions. Basing their analysis on the method of harmonic balance, they obtained superharmonic and subharmonic solutions. They also conducted an experiment; the results are basically in agreement with the analysis, the response having the various different frequencies predicted by the analysis.

$\mathrm{Mei}^{7}$ used a finite-element method to analyze the large-amplitude, free oscillations of beams, but he did not consider modal couplings. His results agree closely with the experimental data of Ray and Bert ${ }^{8}$ for beams having hinged ends.

The same basic approach of expressing the deflection as an expansion in terms of the linear modes was used by Chu and Herrmann ${ }^{9}$ and Chiang and Chen ${ }^{10}$ in a nonlinear analysis of the vibration of plates. Using the dynamic form of the von Karman and Berger equations, respectively, they formulated the problem in such a way as to allow for modal couplings, but in both studies the coupling was neglected. Apparently an analysis, similar to that for beams, which includes modal coupling, has not yet been done for plates.

Recently, Nayfeh ${ }^{11}$ and Atluri ${ }^{12}$ applied versions of the method of multiple scales, in place of harmonic balance, to the study of beam vibrations. Three versions 
of the method of multiple scales are discussed in detail in chapter six of the text by Nayfeh. ${ }^{13}$ Using the general version, Nayfeh considered a beam with slowly varying properties along its length. He did not consider modal couplings. Atluri used the derivative expansion version to study the free, undamped vibrations of a beam with hinged ends, but without axial force. Longitudinal and rotary inertia effects were included, and modal couplings were considered.

In the present paper, we describe a general procedure that can be used to solve the nonlinear equations governing the large-amplitude oscillations of structural elements. The deflection is represented by an expansion in terms of the linear, free-oscillation modes; modal interactions are given strong consideration. Emphasis is placed on determining the response to a harmonic excitation; all frequencies are considered, but special emphasis is placed on the case when the excitation frequency is near a natural frequency. We specialize the general procedure to beams and include the effects of viscous damping and axial force. Finally, we present a numerical example in which there is strong modal coupling. The present results show some interesting features of large-amplitude beam oscillations which, to the author's knowledge, have never been displayed before.

\section{DESCRIPTION OF THE GENERAL METHOD}

We consider systems which are governed by equations of the form

$$
\frac{\partial^{2} w}{\partial t^{2}}+L(w)=\epsilon[D(w)+N(w)+p(\mathbf{r}, t)],
$$

where $L$ is a linear spatial operator, $D$ is the damping operator, $N$ is a nonlinear spatial operator, $p$ is the forcing function, $r$ is the position vector, and $\epsilon$ is a small parameter which appears when the equation is written in terms of dimensionless variables; $N(w)$ is the term which accounts for mid-plane stretching. In addition to Eq. 1a, $w$ must satisfy initial conditions and a set of homogeneous boundary conditions. of the form

$$
B(w)=0 \text { on } \Gamma \text {, }
$$

where $B$ is a linear, spatial operator and $\Gamma$ is the boundary of the spatial domain.

As $\epsilon \rightarrow 0$, Eq. 1a reduces to

$$
\frac{\partial^{2} w}{\partial t^{2}}+L(w)=0
$$

Assuming separation of variables, we put

$$
w=\sum_{m} u_{m}(t) \phi_{m}(\mathbf{r})
$$

and obtain from Eqs. 2 and $1 b$

$$
\frac{d^{2} u_{m}}{d t^{2}}+\omega_{m}^{2} u_{m}=0
$$

and

$$
L\left(\phi_{m}\right)-\omega_{m}^{2} \phi_{m}=0,
$$

where

$$
B\left(\phi_{m}\right)=0 \text { on } \Gamma \text {. }
$$

The summation indicated in Eq. 3 may be a multiple summation depending on the number of independent spatial variables. The eigenfunctions $\phi_{m}$ and the eigenvalues $\omega_{m}$ obtained from Eqs. 5 are the linear, free-oscillation modes and the natural frequencies, respectively. Determination of $\phi_{m}$ and $\omega_{m}$ can be accomplished analytically for some cases of interest, but generally one should expect to obtain them with a numerical procedure.

For the nonlinear problem, we also express the solution in the form given in Eq. 3. The $\phi_{m}$ are the solutions of Eqs. 5, but the $u_{m}$ are not solutions of Eq. 4, as in the linear problem. Instead, the governing equations for the $u_{m}$ are a set of coupled, nonlinear, secondorder, ordinary differential equations which are obtained by substituting Eq. 3 into Eq. 1a, multiplying by $\phi_{n}$ (where $n=1,2, \ldots$ ) and the weighting function, and integrating over the spatial domain. The equations which result are of the form:

$$
\begin{array}{r}
\frac{d^{2} u_{n}}{d t^{2}}+\omega_{n}^{2} u_{n}=\epsilon\left[-2 \sum_{m} c_{m n} \frac{d u_{m}}{d t}+f_{n}\left(u_{1}, u_{2}, \ldots\right)\right. \\
\left.+P_{n}(t)\right], \text { for } n=1,2, \ldots .
\end{array}
$$

We simplify this equation by considering the damping matrix $c_{m n}$ to be diagonal (i.e., modal damping), the forcing function to be harmonic, and $f_{n}$ to be cubic in form. Hence, we put

$$
\begin{aligned}
& \quad c_{m n}=\delta_{m n} c_{n}, \\
& P_{n}(t)=F_{n} \cos \lambda t, \\
& \text { and } \\
& \qquad f_{n}=\sum_{m, p, q} \alpha_{n m p q} u_{m} u_{p} u_{q} \cdot \\
& \text { Then, Eqs. } 6 \text { become } \\
& \frac{d^{2} u_{n}}{d t^{2}}+\omega_{n}^{2} u_{n}=\epsilon\left(-2 c_{n} \frac{d u_{n}}{d t}+\sum_{m, p, q} \alpha_{n m p q} u_{m} u_{p} u_{q}+F_{n} \cos \lambda t\right)
\end{aligned}
$$

Equations 7 are similar to the Duffing equation and, as in the case of the Duffing equation, the straightforward expansion contains secular terms of the form $t^{n}$ $\exp (i \omega t)$ and, hence, is not uniformly valid. The method of multiple scales can be used to construct a uniformly valid, asymptotic expansion.

According to this method, we assume that each $u_{n}$ is a function of different time scales which are defined by

$$
T_{n}=\epsilon^{n} t
$$

and can be expanded in the form

$$
u_{n}(t ; \epsilon) \sim \sum_{j=0} \epsilon^{j} u_{n j}\left(T_{0}, T_{1}, \ldots\right), \text { for } n=1,2, \ldots .
$$

Derivatives with respect to time transform according to

$$
\frac{d}{d t}=D_{0}+\epsilon D_{1}+\cdots,
$$

and

$$
\frac{d^{2}}{d t^{2}}=D_{0}^{2}+2 \epsilon D_{0} D_{1}+\cdots,
$$

Substituting Eqs. 8 into Eqs. 7 and equating coefficients 
of like powers of $\epsilon$, we obtain:

Order $\epsilon^{0}$ :

$$
D_{0}^{2} u_{n 0}+\omega_{n}^{2} u_{n 0}=0, \text { for } n=1,2, \ldots \text {. }
$$

Order $\epsilon$ :

$$
\begin{aligned}
D_{0}^{2} u_{n 1}+\omega_{n}^{2} u_{n 1}= & -2 D_{0} D_{1} u_{n 0}-2 c_{n} D_{0} u_{n 0}+F_{n} \cos \lambda T_{0} \\
& +\sum_{m, p, q} \alpha_{n m p q} u_{m 0} u_{p 0} u_{q 0}, \text { for } n=1,2, \ldots
\end{aligned}
$$

etc.

It is convenient to write the solution to Eqs. 9 as follows:

$$
u_{n 0}=A_{n}\left(T_{1}, T_{2}, \ldots\right) \exp \left(i \omega_{n} T_{0}\right)+c c, \text { for } n=1,2, \ldots,
$$

where $c c$ represents the complex conjugate of the term(s) to the left. At this point, the $A_{n}$ are unknown. They are determined from the solvability conditions (i.e., elimination of secular terms) at the next level of approximation. Putting Eqs. 11 into Eqs. 10 leads to

$$
\begin{aligned}
D_{0}^{2} u_{n 1}+\omega_{n}^{2} u_{n 1}= & -i 2 \omega_{n}\left(D_{1} A_{n}+c_{n} A_{n}\right) \exp \left(i \omega_{n} T_{0}\right) \\
& +\frac{1}{2} F_{n} \exp \left(i \lambda T_{0}\right) \\
& +\sum_{m, p, q} \alpha_{n m p q}\left\{A_{m} A_{p} A_{q} \exp \left[i\left(\omega_{m}+\omega_{p}+\omega_{q}\right) T_{0}\right]\right. \\
& +A_{m} A_{p} \bar{A}_{q} \exp \left[i\left(\omega_{m}+\omega_{p}-\omega_{q}\right) T_{0}\right] \\
& +A_{m} \bar{A}_{p} A_{q} \exp \left[i\left(\omega_{m}-\omega_{p}+\omega_{q}\right) T_{0}\right] \\
& \left.+A_{m} \bar{A}_{p} \bar{A}_{q} \exp \left[i\left(\omega_{m}-\omega_{p}-\omega_{q}\right) T_{0}\right]\right\}+c c,
\end{aligned}
$$$$
\text { for } n=1,2, \ldots \text {. }
$$

In order to eliminate the secular terms from $u_{n 1}$, the $A_{m}$ must be chosen so that the coefficient of $\exp \left(i \omega_{n} T_{0}\right)$ is zero. This coefficient will contain $F_{n}$ when $\lambda$ is near $\omega_{n}$ as well as the nonlinear terms associated with any combination of $\omega_{m}, \omega_{p}, \omega_{q}$ which nearly equals $\omega_{n}$, namely combinations of the form

$$
\begin{aligned}
& \omega_{n} \approx \omega_{m}+\omega_{p}+\omega_{q}, \\
& \text { or } \omega_{n} \approx \omega_{m}+\omega_{p}-\omega_{q}, \\
& \text { or } \\
& \omega_{n} \approx \omega_{n}-\omega_{p}-\omega_{q} . \\
& \text { The natural frequencies being commensurable is re- } \\
& \text { ferred to as internal resonance. }
\end{aligned}
$$

As an example, we consider the case in which

$$
\omega_{4}=\omega_{3}+\omega_{2}+\omega_{1}+\epsilon \sigma_{1} \text { and } \lambda=\omega_{4}+\epsilon \sigma_{2} .
$$

The detuning parameters $\sigma_{1}$ and $\sigma_{2}$ are introduced in order to describe the nearness of the approximations conveniently. Then, it follows from Eqs. 12 that secular terms will not appear in the $u_{n 1}$, for $n=1,2$, and 3 , if

$$
\begin{array}{r}
-i 2 \omega_{n}\left(D_{1} A_{n}+c_{n} A_{n}\right)+A_{n} \sum_{j} \gamma_{n j} A_{j} \bar{A}_{j}+\alpha_{n} A_{4} \bar{A}_{k} \bar{A}_{l} \\
\times \exp \left(i \sigma_{1} T_{1}\right)=0 \text { for } n=1,2 \text {, and } 3,
\end{array}
$$

$k, l$, and $n$ are different; each is limited to 1,2 , and 3 . The $\gamma_{n j}$ and $\alpha_{n}$ are determined by performing the summations indicated in Eq. 12. For $n=4$, secular terms are eliminated if

$$
\begin{array}{r}
-2 i \omega_{4}\left(D_{1} A_{4}+c_{4} A_{4}\right)+A_{4} \sum_{j} \gamma_{4 j} A_{j} \bar{A}_{j}+\alpha_{4} A_{3} A_{2} A_{1} \\
\times \exp \left(-i \sigma_{1} T_{1}\right)+\frac{1}{2} F_{4} \exp \left(i \sigma_{2} T_{1}\right)=0
\end{array}
$$

and for $n>4$, secular terms are eliminated if

$$
-2 i \omega_{n}\left(D_{1} A_{n}+c_{n} A_{n}\right)+A_{n} \sum_{j} \gamma_{n j} A_{j} \bar{A}_{j}=0
$$

It is generally more convenient to consider the equations governing the interactions of the amplitudes and phases of the interacting modes. Consequently, we put

$$
A_{n}=\frac{1}{2} a_{n}\left(T_{1}\right) \exp \left[i \beta_{n}\left(T_{1}\right)\right], \text { for } n=1,2, \ldots \text {, }
$$

where $a_{n}$ and $\beta_{n}$ are real. Because we are only interested in the first term of the expansions for $u_{n}$, all $T_{n}$ for $n$ greater than 1 are considered constants. Substituting Eqs. 14 into Eqs. 13 and separating the result into real and imaginary parts, we obtain

$$
\omega_{n} a_{n}^{\prime}=-c_{n} \omega_{n} a_{n}+\frac{1}{8} \alpha_{n} a_{4} a_{k} a_{l} \sin \mu_{1},
$$

and

$$
\omega_{n} a_{n} \beta_{n}^{\prime}=-\frac{1}{8}\left(a_{n} \sum_{j} \gamma_{n j} a_{j}^{2}+\alpha_{n} a_{4} a_{k} a_{l} \cos \mu_{1}\right)
$$

for $n=1,2$, and $3 ; k, l$, and $n$ are different and limited to 1,2 , and 3 . The primes denote differentiation with respect to $T_{1}$, and

$$
\begin{aligned}
& \mu_{1}=\sigma_{1} T_{1}+\beta_{4}-\beta_{3}-\beta_{2}-\beta_{1}, \\
& \omega_{4} a_{4}^{\prime}=-c_{4} \omega_{4} a_{4}-\frac{1}{8} \alpha_{4} a_{3} a_{2} a_{1} \sin \mu_{1}+\frac{1}{2} F_{4} \sin \mu_{2}=0,
\end{aligned}
$$

and

$$
\omega_{4} a_{4} \beta_{4}^{\prime}=-\frac{1}{8}\left(a_{4} \sum_{j} \gamma_{4 j} a_{j}^{2}+\alpha_{4} a_{3} a_{2} a_{1} \cos \mu_{1}\right)-\frac{1}{2} F_{4} \cos \mu_{2},
$$

$\mu_{2}=\sigma_{2} T_{1}-\beta_{4}$

For $n>4$, we have

$$
\omega_{n} a_{n}^{\prime}=-c_{n} \omega_{n} a_{n}
$$

and

$$
\omega_{n} a_{n} \beta_{n}^{\prime}=-\frac{1}{8} a_{n} \sum_{j} \gamma_{n j} a_{j}^{2} .
$$

From these equations, we can determine a steadystate solution (constant $a_{n}$ and $\mu_{n}$ ) in which the amplitude and phases are functions of the detuning parameters $\sigma_{1}$ and $\sigma_{2}$, the amplitude of the excitation $F_{4}$ and the damping coefficients $c_{n}$. It follows immediately that in the first approximation the response is composed of that mode having a natural frequency near the excitation frequency and possibly all the other modes involved in the internal resonance; the remaining modes are damped out. If other modes are excited, then the response contains several harmonics, and some of them may be stronger than the one near the excitation frequency.

There are other possible combinations of natural frequencies which can produce internal resonances, and these situations are treated in a similar way.

There are resonant situations that can arise when $\lambda$ is not near any $\omega_{n}$. To consider these situations, we let $F$ be $0\left(\epsilon^{-1}\right)$ and proceed as above. Now, however, the first terms in the expansions for the $u_{n}$ are of the 


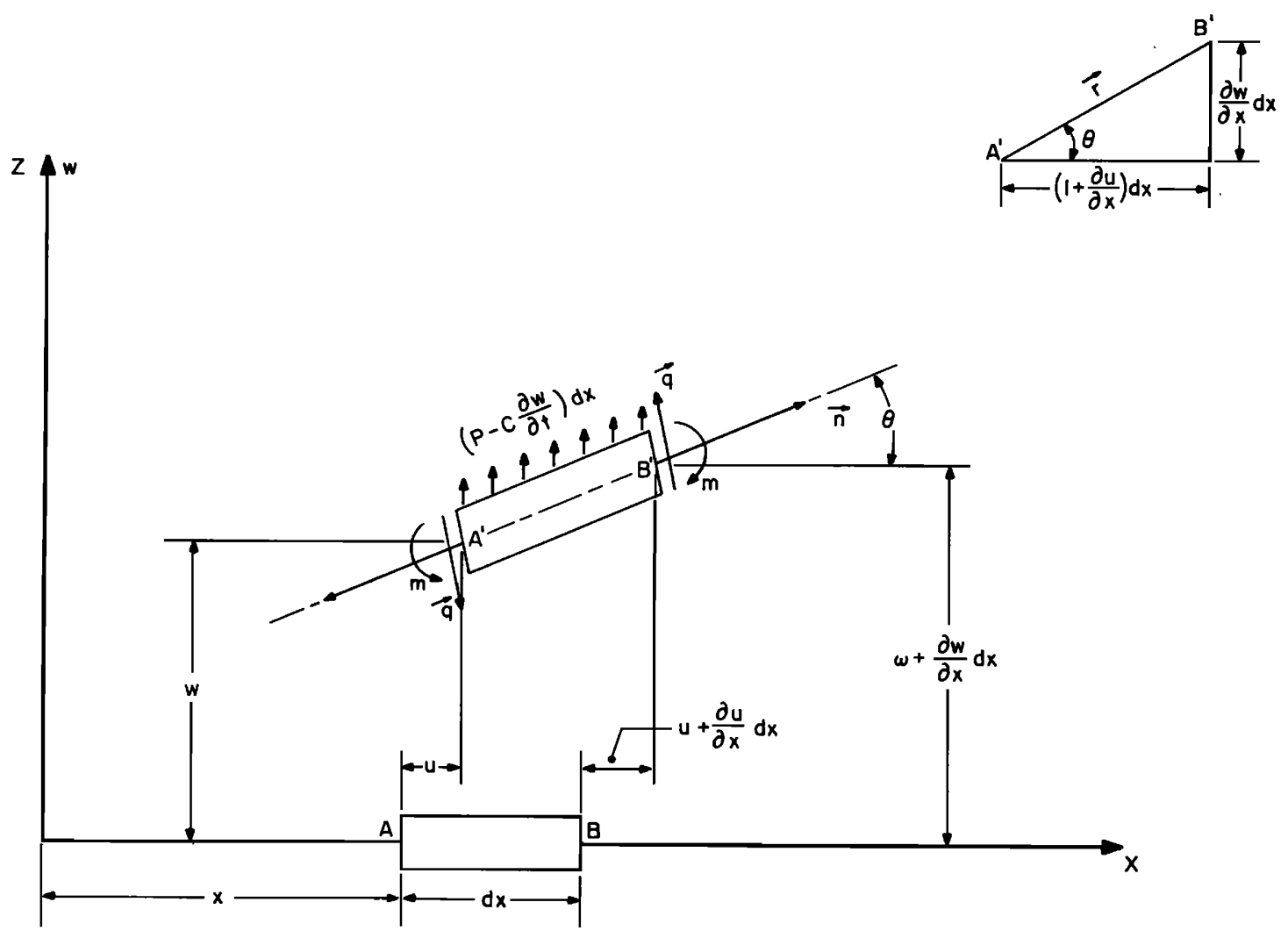

FIG. 1. An Element of the Beam.

form

$$
u_{n 0}=A_{n}\left(T_{1}\right) \exp \left(i \omega_{n} T_{0}\right)+\frac{1}{2} F_{n}\left(\omega_{n}^{2}-\lambda^{2}\right)^{-1} \exp \left(i \lambda T_{0}\right)+c c .
$$

The right-hand side of the governing equations for the $u_{n 1}$ will contain (among others) factors of the form

$$
\begin{gathered}
\exp \left\{i \left[\left(\omega_{m}+\omega_{p}+\omega_{q}\right),\left(\omega_{m}-\omega_{p}+\omega_{q}\right),\left(\omega_{m}-\omega_{p}-\omega_{q}\right),(3 \lambda),\right.\right. \\
\left.\left.\left(2 \lambda-\omega_{m}\right),\left(\lambda-\omega_{m}-\omega_{k}\right),\left(\lambda-\omega_{m}+\omega_{k}\right)\right]\right\} .
\end{gathered}
$$

Any situation in which there is a combination of frequencies that equals, or nearly equals $\omega_{n}$, involves modal interactions. This case contains the so-called superharmonic and subharmonic responses.

Finally, if none of the above situations arise, then to the first approximation the steady-state solution consists only of the mode being excited.

\section{STABILITY OF THE VARIOUS SOLUTIONS}

Generally, not all the steady-state solutions of Eqs. 15 are stable. To study the stability, we determine the response of the system caused by an infinitesimal perturbation away from a steady-state solution. We put

$$
a_{n}=\tilde{a}_{n}+\Delta a_{n},
$$

and

$$
\mu_{n}=\tilde{\mu}_{n}+\Delta \mu_{n},
$$

where the tilde indicates the steady-state solution. When Eqs. 16 are substituted into Eqs. 15 and only linear terms in the perturbations retained, the result is a linear set of first-order equations with constant coefficients governing the $\Delta a_{n}$ and $\Delta \mu_{n}$. These equations have a solution proportional to $\exp \left(m T_{1}\right)$ where $m$ is an eigenvalue of the coefficient matrix. If none of the $m$ 's has a positive real part, the motion is considered stable.

In previous stability studies, the small disturbances were put into Eqs. 7 instead of Eqs. 15. This leads to coupled equations of the Mathieu type and generally requires more effort to determine the stability.

\section{FORCED OSCILLATIONS OF PRISMATIC BEAMS}

Referring to Fig. 1, we write the three pertinent equations of motion as follows:

$x$-momentum:

$$
\frac{\partial}{\partial x^{\prime}}\left(n^{\prime} \cos \theta-q^{\prime} \sin \theta\right)=\rho A \frac{\partial^{2} u^{\prime}}{\partial t^{2}}
$$

$z$-momentum:

$$
\frac{\partial}{\partial x^{\prime}}\left(n^{\prime} \sin \theta+q^{\prime} \cos \theta\right)+P^{\prime}-c^{\prime} \frac{\partial w^{\prime}}{\partial t^{\prime}}=\rho A \frac{\partial^{2} w^{\prime}}{\partial t^{2}} ;
$$

moment of momentum:

$$
\begin{aligned}
-\frac{\partial m^{\prime}}{\partial x^{\prime}}+n^{\prime} & {\left[\left(1+\frac{\partial u^{\prime}}{\partial x^{\prime}}\right) \sin \theta-\frac{\partial w^{\prime}}{\partial x^{\prime}} \cos \theta\right] } \\
+q^{\prime} & {\left[\left(1+\frac{\partial u^{\prime}}{\partial x^{\prime}}\right) \cos \theta+\frac{\partial w^{\prime}}{\partial x^{\prime}} \sin \theta\right]=\rho I \frac{\partial^{2} \theta}{\partial t^{\prime 2}} . }
\end{aligned}
$$

In the usual way, we assume that the loads are related to the displacements as follows:

$$
n^{\prime}=E A e^{\prime} \text { and } m^{\prime}=-E I \frac{\partial \theta}{\partial x},
$$

where

$$
e^{\prime}=S-1
$$

and 


$$
S=\left[\left(1+\frac{\partial u}{\partial x}\right)^{2}+\left(\frac{\partial w}{\partial x}\right)^{2}\right]^{1 / 2}
$$

Here, $E$ is the elastic modulus, $A$ and $I$ are the area and the moment of inertia of the cross section, and $\rho$ is the density of the beam.

It is convenient to re-write the equations in terms of dimensionless variables (without primes) and in the process to introduce a dimensionless parameter which can be useful in constructing an asymptotic expansion of the solution. Using Eqs. 18 and combining Eqs. 17b and $17 \mathrm{c}$ to eliminate $q^{\prime}$, we obtain

$$
\frac{\partial e}{\partial x}=0(\epsilon)
$$

and

$$
\frac{\partial^{4} w}{\partial x^{4}}+\frac{\partial^{2} w}{\partial t^{2}}=\left[\frac{\partial}{\partial x}\left(e \frac{\partial w}{\partial x}\right)+p-2 c \frac{\partial w}{\partial t}\right],
$$

where

$$
\begin{aligned}
& x^{\prime}=x L, t^{\prime}=\left(\rho L^{4} / E r^{2}\right)^{1 / 2} t, \epsilon=r^{2} / L^{2} . \\
& w^{\prime}=r^{2} w / L, u^{\prime}=\epsilon r^{2} u / L, \quad p=p^{\prime} L^{7} /\left(r^{6} E A\right)
\end{aligned}
$$

and

$$
c=c^{\prime} L^{4} /\left[2 \gamma^{3} A(\rho E)^{1 / 2}\right]
$$

Here, $r$ is the radius of gyration of the cross-section area, and $L$, the characteristic length, may be the actual length of the beam or a wavelength of a (linear) transverse oscillation.

Integrating Eq. 19a leads to

$$
e=\frac{\partial u}{\partial x}+\frac{1}{2}\left(\frac{\partial w}{\partial x}\right)^{2}=H(t)
$$

and

$$
u=G(t)+x H(\dot{t})-\frac{1}{2} \int_{0}^{x}\left(\frac{\partial w}{\partial x}\right)^{2} d x,
$$

where $G$ and $H$ are arbitrary functions. For boundary conditions, we use

$$
u(0, t)=0 \quad \text { and } \delta u(l, t)+e(l, t)=0 .
$$

Here, the constant $\delta$ depends on how the beam is supported; $\delta$ is zero for no restraint and infinity for a rigid restraint. It follows that

$$
G(t) \equiv 0, H(t) \equiv \frac{\delta}{2(1+\delta l)} \int_{0}^{l}\left(\frac{\partial w}{\partial x}\right)^{2} d x,
$$

and

$$
\frac{\partial^{4} w}{\partial x^{4}}+\frac{\partial^{2} w}{\partial t^{2}}=\epsilon\left(H \frac{\partial^{2} w}{\partial x^{2}}+p-2 c \frac{\partial w}{\partial t}\right) .
$$

This is the form of Eq. 1a which applies to beams.

Following the general procedure, we put

$$
w(x, t)=\sum_{m} u_{m}(t) \phi_{m}(x),
$$

substitute into Eq. 20, and obtain

$$
\frac{d^{4} \phi_{m}}{d x^{4}}-\omega_{m}^{2} \phi_{m}=0
$$

and

$$
\begin{aligned}
\frac{d^{2} u_{n}}{d t^{2}}+\omega_{n}^{2} u_{n}= & \epsilon\left(-2 c_{n} \frac{d u_{n}}{d t}+F_{n} \cos \lambda t\right. \\
& \left.+\nu \sum_{m, p, Q} \alpha_{n m p q} u_{m} u_{p} u_{q}\right)
\end{aligned}
$$

where

$$
\nu=\frac{\delta}{2(1+\delta l)},
$$

and

$$
\alpha_{n m p q}=\left[\int_{0}^{l}\left(\phi_{n} \frac{d^{2} \phi_{q}}{d x^{2}} d x\right]\left[\int_{0}^{l}\left(\frac{d \phi_{m}}{d x} \frac{d \phi_{p}}{d x}\right) d x\right] .\right.
$$

Equations 22 are of the same form as Eqs. 7, and the solution follows the procedure which was described in the previous section. In the next section we consider a numerical example.

\section{NUMERICAL EXAMPLE}

We begin by considering a beam with one end hinged and the other end clamped. The boundary conditions lead to

$$
\phi_{m}(0)=0, \frac{d^{2} \phi_{m}}{d x^{2}}(0)=0, \quad \phi_{m}(l)=0
$$

and

$$
\frac{d \phi_{m}}{d x}(l)=0
$$

The solution of Eq. 21 which satisfies these conditions is

$$
\phi_{n}=E_{n}\left(\sin \alpha_{n} x-R_{n} \sinh \alpha_{n} x\right),
$$

where

$$
\begin{aligned}
& E_{n}=\left[\frac{1}{2} l\left(1-R_{n}^{2}\right)+\left(R_{n}^{2} \sinh 2 \alpha_{n} l-\sin 2 \alpha_{n} l\right) / 4 \alpha_{n}\right]^{-1 / 2}, \\
& R_{n}=\sin \alpha_{n} l / \sinh \alpha_{n} l,
\end{aligned}
$$

and the $\alpha_{n}$ are the roots of

$$
\tan \alpha_{n} l=\tanh \alpha_{n} l
$$

The first five roots and frequencies are (for $l=1$ )

$$
\begin{aligned}
& \alpha_{1} l=3.927 \quad \text { and } \omega_{1}=15.421, \\
& \alpha_{2} l=7.069 \text { and } \omega_{2}=49.970, \\
& \alpha_{9} l=10.210 \text { and } \omega_{3}=104.24, \\
& \alpha_{4} l=13.352 \text { and } \omega_{4}=178.28,
\end{aligned}
$$

and

$$
\alpha_{5} l=16.493 \text { and } \omega_{5}=272.02 .
$$

Note that $\omega_{2}$ and $\omega_{1}$ are nearly in the ratio of three to one. There may be more commensurable combinations of the $\omega_{n}$, but we restrict our attention here to this case. To express the nearness of $\omega_{2}$ to $3 \omega_{1}$, we introduce a detuning parameter $\sigma_{1}$ as follows:

$$
\omega_{2}=3 \omega_{1}\left(1+\epsilon \sigma_{1}\right),
$$

where

$$
\epsilon \sigma_{1}=0.0801 \text {. }
$$

There are two cases of interest: (A) $\lambda$ near $\omega_{1}$ and 


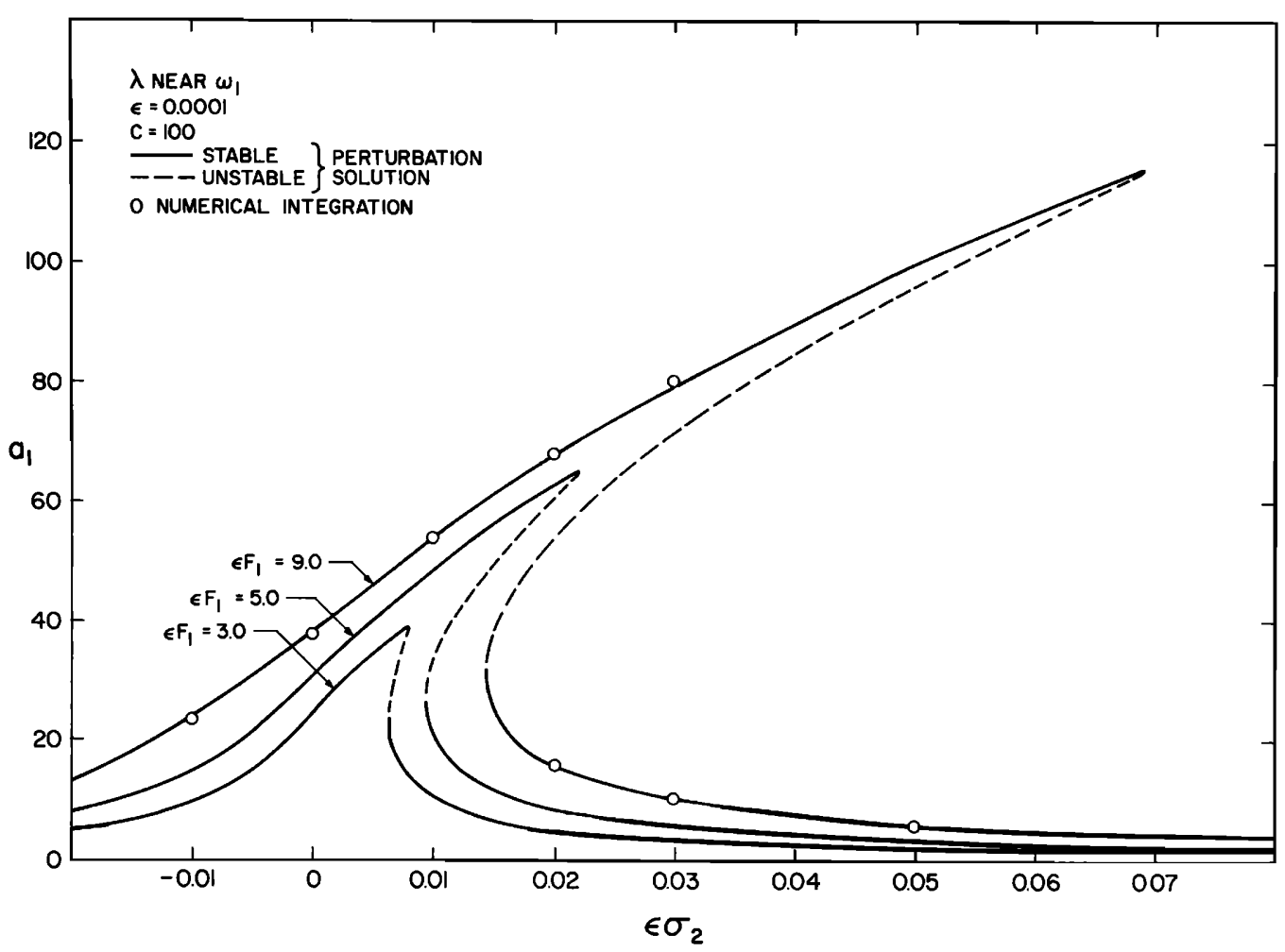

(a)

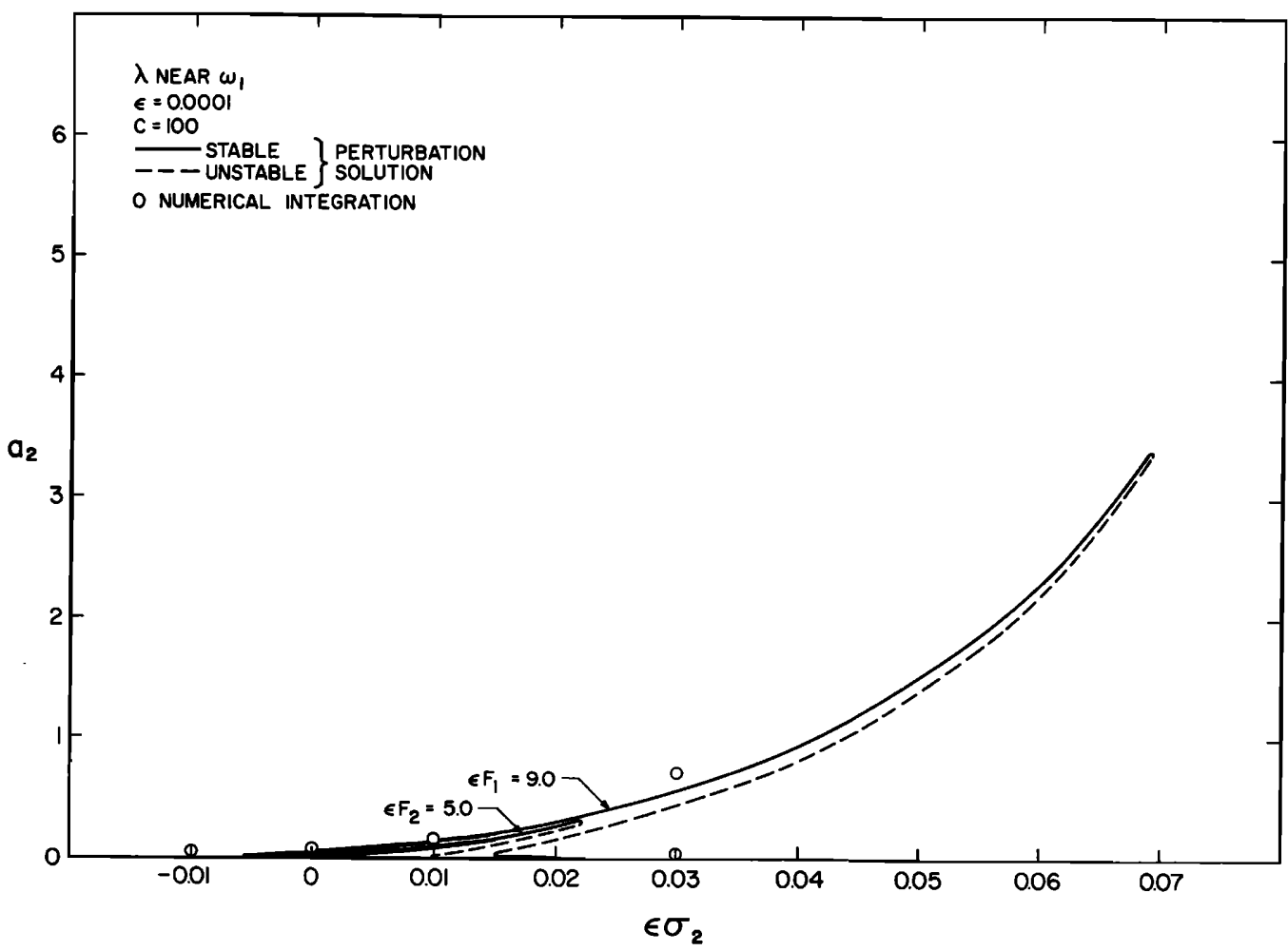

(b)

FIG. 2. (a) Variation of amplitude $a_{1}$ of first mode with the excitation frequency $\sigma_{2}$. (b) Variation of amplitude $a_{2}$ of second mode with $\sigma_{2}$.

(B) $\lambda$ near $\omega_{2}$. To express the nearness of $\lambda$ to the $\omega_{n}$, we introduce a second detuning parameter $\sigma_{2}$ as follows:

$$
\lambda=\omega_{n}\left(1+\epsilon \sigma_{2}\right), \quad \text { for } n=1 \text { and } 2 .
$$

For convenience, we put

$$
A_{n}=\frac{1}{2} a_{n} \exp \left(i \omega_{1} \beta_{n}\right) \text { for } n=1,2, \ldots \text {. }
$$

Then following the general procedure, we obtain the following from Eqs. 22:

$$
\begin{aligned}
& \text { A. } \lambda \text { near } \omega_{1} \\
& \text { For } n=1, \\
& \omega_{1}\left(a_{1}^{\prime}+c_{1} a_{1}\right)-\frac{1}{8} \nu q_{3} a_{1}^{2} a_{2} \sin \mu_{1}-\frac{1}{2} F_{1} \sin \mu_{2}=0
\end{aligned}
$$


and

$$
\begin{aligned}
& \omega_{1}^{2} a_{1} \beta_{1}^{\prime}+\frac{1}{8} \nu\left(q_{1} a_{1}^{3}+q_{2} a_{2}^{2} a_{1}+q_{3} a_{1}^{2} a_{2} \cos \mu_{1}\right) \\
&+a_{1} \sum_{j=3}^{\infty} \gamma_{1 j} a_{j}^{2}+\frac{1}{2} F_{1} \cos \mu_{2}=0 .
\end{aligned}
$$

For $n=2$,

$$
\omega_{2}\left(a_{2}^{\prime}+c_{2} a_{2}\right)+\frac{1}{8} \nu q_{6} a_{1}^{3} \sin \mu_{1}=0
$$

and

$$
\begin{aligned}
\omega_{1} \omega_{2} a_{2} \beta_{2}^{\prime}+\frac{1}{8} \nu\left(q_{4} a_{2}^{3}+q_{5} a_{1}^{2} a_{2}+q_{6} a_{1}^{3} \cos \mu_{1}\right) \\
+a_{2} \sum_{j=3}^{\infty} \gamma_{2 j} a_{j}^{2}=0 .
\end{aligned}
$$

For $n>2$,

$$
a_{n}^{\prime}+c_{n} a_{n}=0
$$

and

$$
\omega_{1} \omega_{n} a_{n} \beta_{n}^{\prime}+a_{n} \sum_{j=1}^{\infty} \gamma_{n j} a_{j}^{2}=0
$$

Here we put

$$
\mu_{1}=\omega_{1}\left(3 \sigma_{1} T_{1}-3 \beta_{1}+\beta_{2}\right) \text { and } \mu_{2}=\omega_{1}\left(\sigma_{2} T_{1}-\beta_{1}\right) .
$$

For the steady-state solution, it follows immediately that $a_{n} \equiv 0$ for $n>2$. However, neither $a_{1}$ nor $a_{2}$ can be zero. Consequently, because of the internal resonance, when $\lambda$ is near $\omega_{1}$, the first and second natural modes appear in the response.

Because $a_{n} \equiv 0$ for $n>2$, the $\gamma_{n j}$ are irrelevant. Using $l=2$, one finds that the remaining coefficients are

$$
\begin{aligned}
& q_{1}=3 \alpha_{1111}=-24.851, \\
& q_{2}=4 \alpha_{1212}+2 \alpha_{1221}=-66.319, \\
& q_{3}=2 \alpha_{1121}+\alpha_{1112}=-9.247, \\
& q_{4}=3 \alpha_{2222}=-345.019, \\
& q_{5}=4 \alpha_{2121}+2 \alpha_{2112}=-66,319,
\end{aligned}
$$

and

$$
q_{6}=\alpha_{2111}=-3.082 .
$$

(Note that in this case $\alpha_{i j k l}=\alpha_{l j k i}=\alpha_{i k f i}$.)

\section{B. $\lambda$ near $\omega_{2}$}

$$
\begin{aligned}
& \text { For } n=1, \\
& \quad \omega_{1}\left(a_{1}^{\prime}+c_{1} a_{1}\right)-\frac{1}{8} \nu q_{3} a_{1}^{2} a_{2} \sin \mu_{1}=0,
\end{aligned}
$$

and

$$
\begin{aligned}
& \omega_{1}^{2} a_{1} \beta_{1}^{\prime}+\frac{1}{8} \nu\left(q_{1} a_{1}^{3}+q_{2} a_{1} a_{2}^{2}+q_{3} a_{1}^{2} a_{2} \cos \mu_{1}\right) \\
& \quad+a_{1} \sum_{j=3}^{\infty} \gamma_{1 j} a_{j}^{2}=0 . \\
& \quad \text { For } n=2, \\
& \quad \omega_{2}\left(a_{2}^{\prime}+c_{2} a_{2}\right)+\frac{1}{8} \nu q_{6} a_{1}^{3} \sin \mu_{1}-\frac{1}{2} F_{2} \sin \mu_{2}=0
\end{aligned}
$$

and

$$
\begin{gathered}
\omega_{1} \omega_{2} a_{2} \beta_{2}^{\prime}+\frac{1}{8} \nu\left(q_{4} a_{2}^{3}+q_{5} a_{1}^{2} a_{2}+q_{6} a_{1}^{3} \cos \mu_{1}\right) \\
+a_{2} \sum_{j=3}^{\infty} \gamma_{2 j} a_{j}^{2}+\frac{1}{2} F_{2} \cos \mu_{2}=0 .
\end{gathered}
$$

For $n>2$,

$$
a_{n}^{\prime}+c_{n} a_{n}=0
$$

and

$$
\omega_{1} \omega_{n} \beta_{n}^{\prime} a_{n}+a_{n} \sum_{j=1}^{\infty} \gamma_{n j} a_{j}^{2}=0
$$

Here $\mu_{1}$ is as defined above, but now

$$
\mu_{2}=\omega_{2} \sigma_{2} T_{1}-\omega_{1} \beta_{2} \text {. }
$$

For the steady-state solution, it follows immediately that for $n>2$

$$
a_{n} \equiv 0 \text {. }
$$

Consequently, the $\gamma_{n j}$ are irrelevant. The $q_{j}$ are the same as in Case A. In this case $a_{1}=0$ is a possible steady-state solution, in contrast to Case A where neither $a_{1}$ nor $a_{2}$ could ever be zero.

For a beam with both ends hinged, there are commensurable natural frequencies, but $\alpha_{i j k l}=0$ for $j \neq k$. For a beam with both ends clamped, the first six natural frequencies, at least, are not commensurable. Consequently, there is no internal resonance phenomenon associated with either case. Only the mode near the exciting frequency is strongly excited, but higher harmonics appear in higher-order terms. The governing equation for each $u_{n}$ is the familiar Duffing equation with a damping and, in some cases, a forcing term. Because there is no strong modal coupling for beams having either clamped or hinged ends, the experimental and analytical results mentioned in the Introduction ought to agree closely.

For some arbitrary values of the forcing amplitude and damping coefficients, we solved Eqs. 23-28 and integrated Eqs. 22 numerically until a steady-state was reached. In Figs. 2(a) and 2(b) the variation of $a_{1}$ and $a_{2}$ with $\sigma_{2}$ is shown for several values of the forcing amplitude when $\lambda$ is near $\omega_{1}$. The result for $a_{1}$ is similar to the solution of the Duffing equation for a hardening spring. Although $a_{2}$ cannot be zero, it is small compared to $a_{1}$, and the response is practically described by the first mode.

In Figs. 3(a) and 3(b) the variation of $a_{1}$ with $F_{1}$ is shown for two values of $\sigma_{2}$. The log-log plot in Fig. 3(b) can be qualitatively compared with the plot of experimental data presented by Jacobsen and van der Hyde. ${ }^{14}$ (The behavior of plates is expected to be similar to that of beams. ) These results suggest that the change in slope found experimentally is a result of restraining the mid-plane movement along the edges of the plate. Such a restraint makes the behavior of plates and beams similar to that of a hardening spring. It should be noted that one would obtain similar results for beams with either clamped or hinged ends. There is no modal interaction for these beams, but the response is governed by the Duffing equation.

In Figs. 4(a) and 4(b), the variation of $a_{1}$ and $a_{2}$ with $\sigma_{2}$ is shown when $\lambda$ is near $\omega_{2}$. When $a_{1}$ is not zero, only the stable portion of the solution is shown. The complete solution (stable as well as unstable regions) when $a_{1}$ is not zero is shown in Fig. 4(b). It is interesting to note that when $a_{1}$ is not zero, it can be as much as six times greater than $a_{2}$. Hence, the response can be dominated 


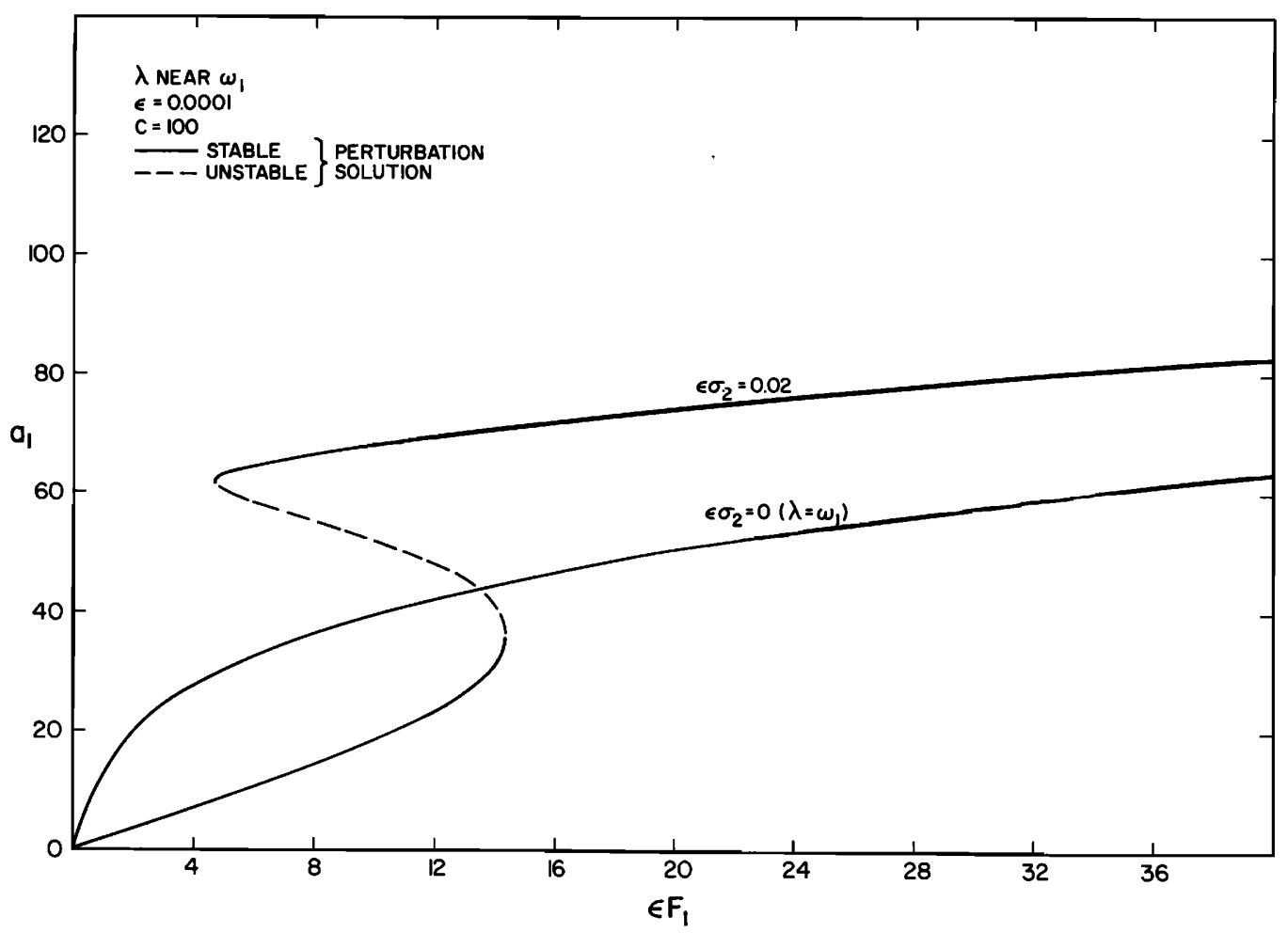

(a)

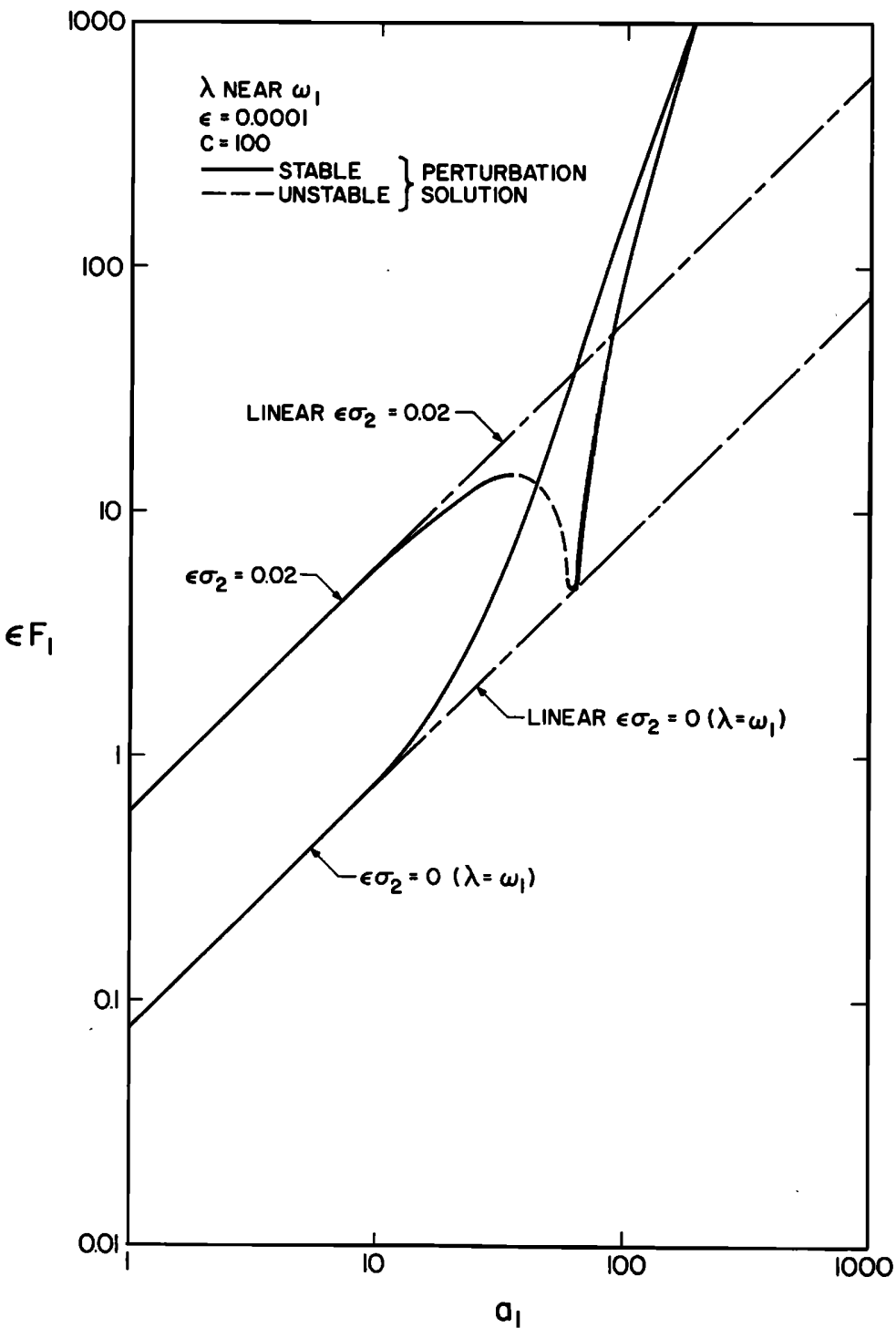

FIG. 3. (a) Variation of $a_{1}$ with $F_{1}$ (amplitude of excitation when frequency near first natural frequency) for the values of $\sigma_{1}$. (b) $L$ og- $\log$ plot of the variation of $a_{1}$ with $F_{1}$ for two values of $\sigma_{2}$.

(b) 


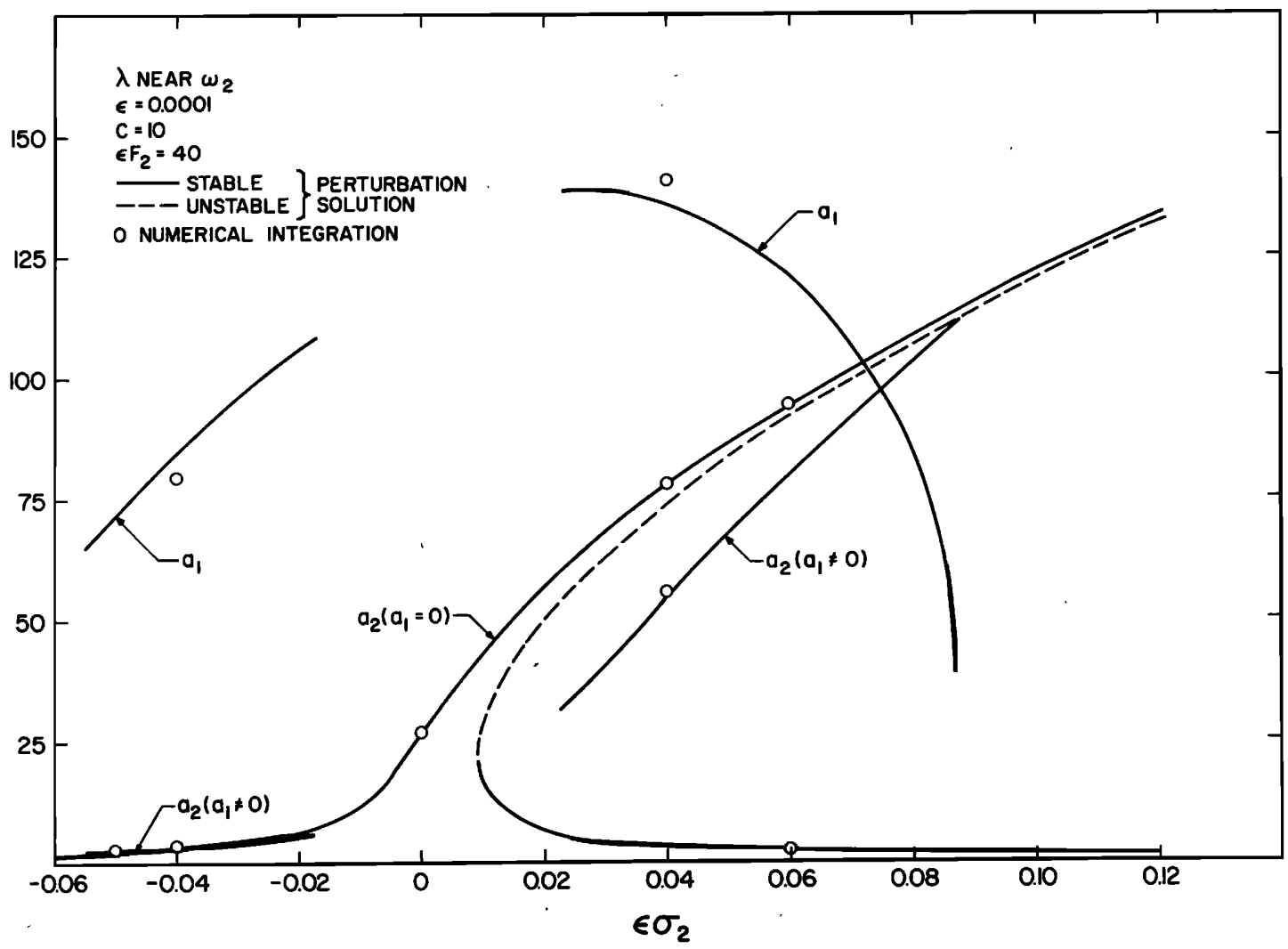

(a)

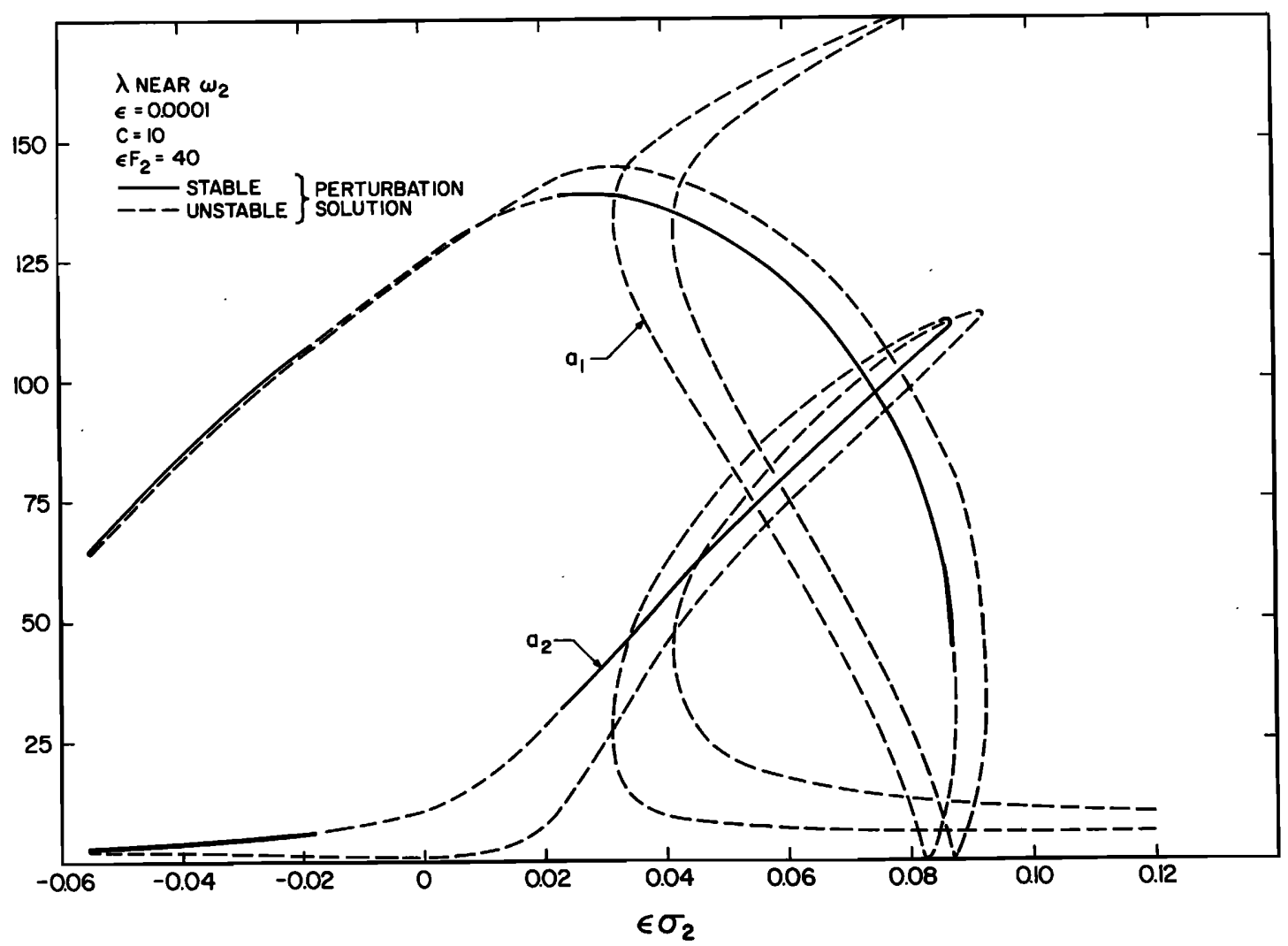

(b)

FIG. 4. (a) Variation of $a_{1}$ and $a_{2}$ with $\sigma_{2}$. (b) Variation of $a_{1}$ and $a_{2}$ with $\sigma_{2}$ when $a_{1}$ is not zero (complete solution).

by the first mode even though the exciting frequency is near the second natural frequency.

The variation of $a_{1}$ and $a_{2}$ with the amplitude of the forcing function is shown in Figs. 5(a) and 5(b). The log-log plots would be similar to Fig. 3(b) with an added branch for the case when $a_{1}$ is not zero.

These results show that a first approximation of the response to a harmonic excitation can involve strong 


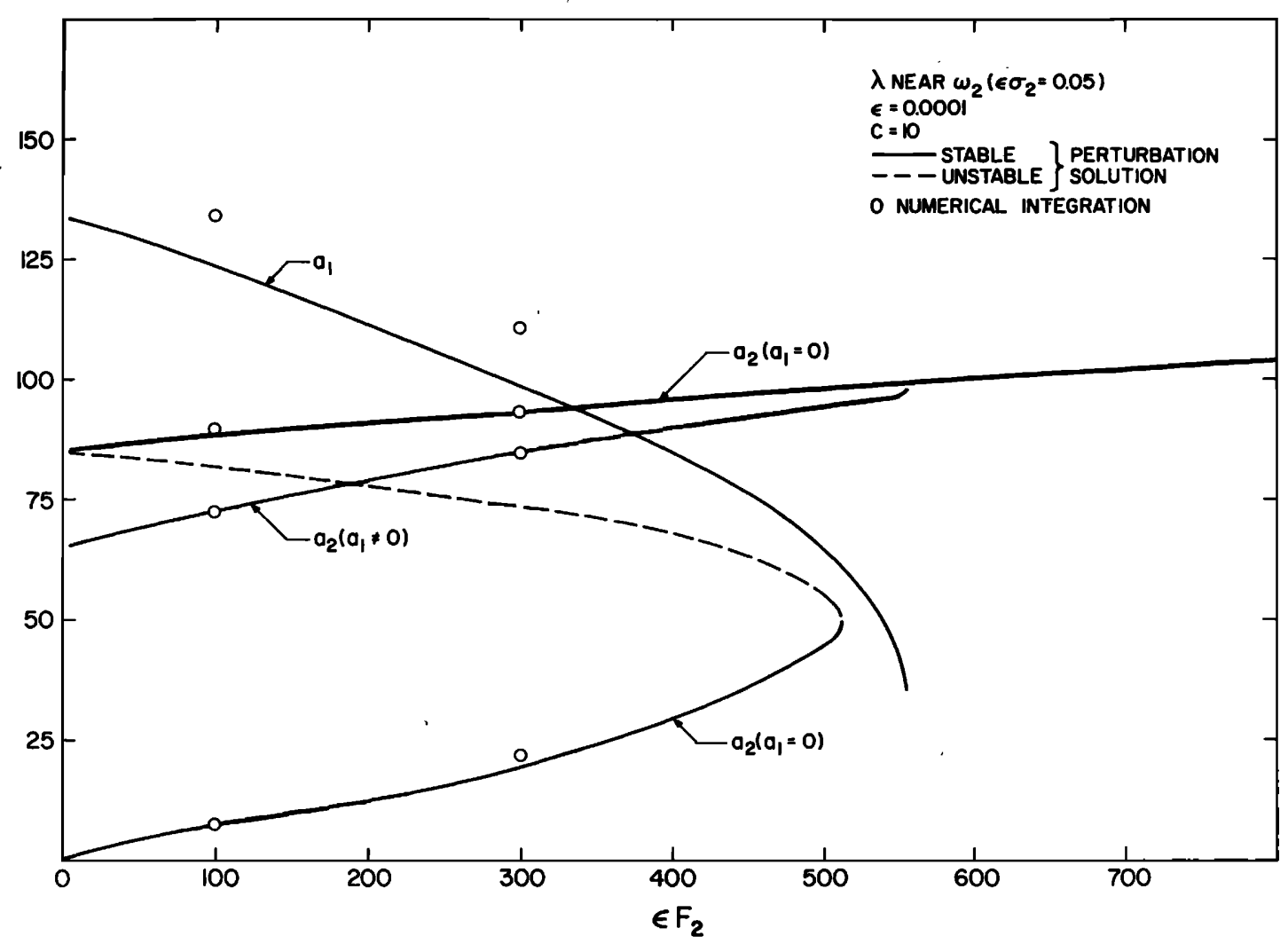

(a)

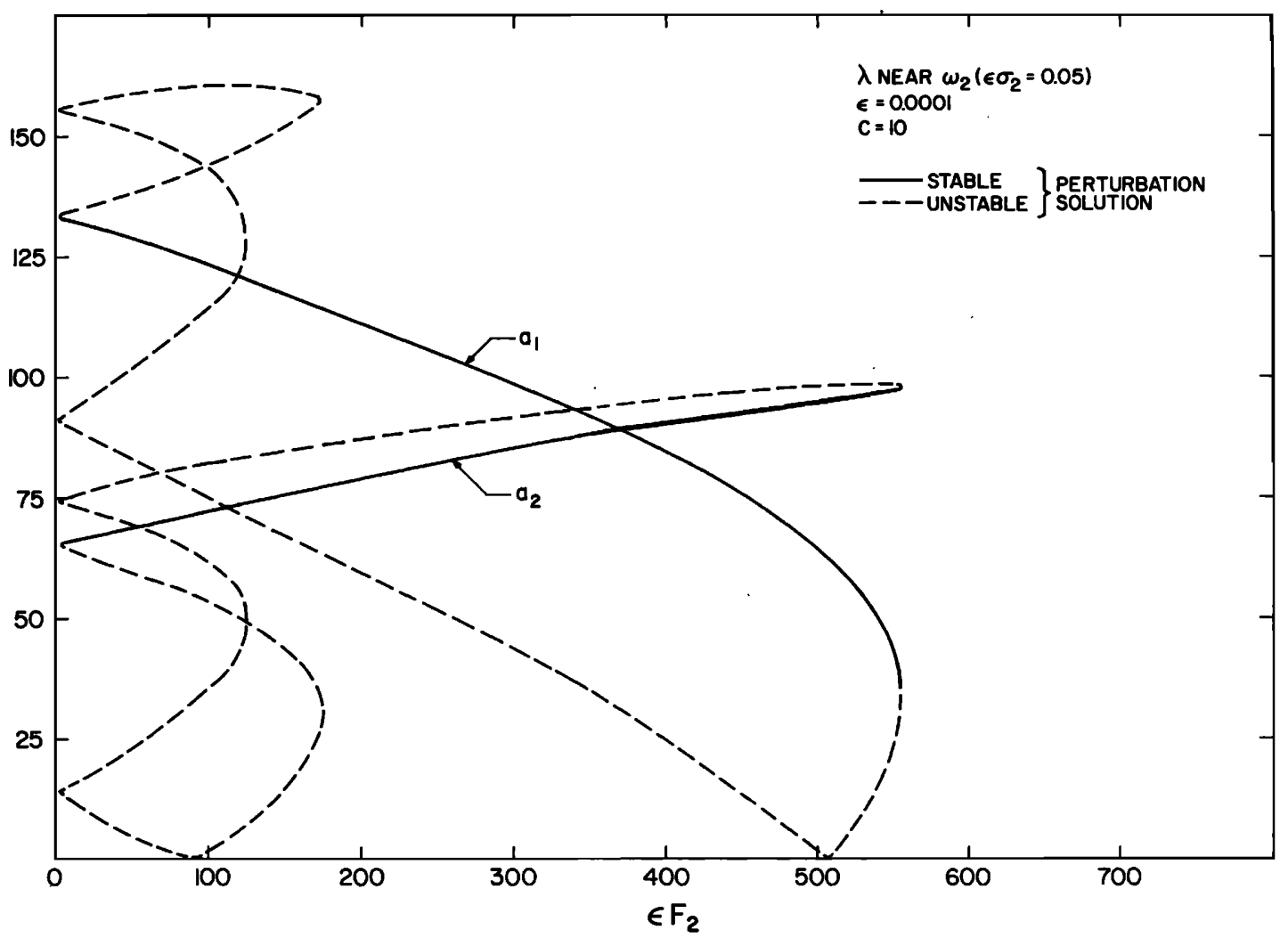

(b)

FIG. 5. (a) Variation of $a_{1}$ and $a_{2}$ with $F_{2}$ (amplitude of excitation when frequency near second natural frequency). (b) Variation of $a_{1}$ and $a_{2}$ with $F_{2}$ when $a_{1}$ is not zero (complete solution).

modal interactions when the natural frequencies are commensurable (internal resonance). In particular, for a beam with one end clamped and the other hinged, it is possible for the response to be dominated by the first free-oscillation mode vibrating at the first natural frequency when the excitation frequency is near the sec- 
ond natural frequency. None of the earlier studies revealed this possibility.

The present results emphasize the need to consider modal interactions in an nonlinear analysis as well as the need for an experiment with a beam having one clamped and one hinged end.

This work was supported by the Structures Division of the Langley Research Center, NASA, under Contract NAS1-10646- 13.

${ }^{1}$ S. Woinowsky-Krieger, "The Effect of an Axial Force on Vibrating Hinged Bars," J. Appl. Mech. 17, 35-37 (1950).

${ }^{2}$ D. Burgreen, "Free Vibrations of a Pin-Ended Column with Constant Distance Between Pin Ends," J. Appl. Mech. 18, 135-139 (1951).

${ }^{3}$ P. H. MacDonald, "Nonlinear Dynamic Coupling in a Beam Vibration," J. Appl. Mech. 22, 573-578 (1955).

${ }^{4} J$. A. Bennett and J. G. Eisley, "A Multiple Degree-of-Freedom Approach to Nonlinear Beam Vibrations, " AIAA J. 8, 734-739 (1970).

5 J. A. Bennett, "Ultraharmonic Motion of a Viscously Damped
Nonlinear Beam," AIAA J. 11, 710-715 (1973).

${ }^{6} \mathrm{~W}$. Y. Tseng and J. Dugundji, "Nonlinear Vibrations of a Buckled Beam under Harmonic Excitation," J. Appl. Mech. 38, 467-476 (1971).

${ }^{7} \mathrm{C}$. Mei, "Nonlinear Vibrations of Beams by Matrix Displacement Methods," AIAA J. 10, 355-357 (1972).

${ }^{8} \mathrm{~J}$. D. Ray and C. W. Bert, "Nonlinear Vibrations of a Beam with Pinned Ends," Trans. ASME, J. Eng. Ind. 91, 997-1004 (1969).

${ }^{9}$ H. N. Chu and G. Hermann, "Influence of Large Amplitude on Free Flexural Vibrations of Rectangular Elastic Plates," J. Appl. Mech. 23, 532-540 (1956).

${ }^{10}$ D. C. Chiang and S. S. H. Chen, "Large Amplitude Vibration of a Circular Plate with Concentric Rigid Mass," J. Appl. Mech. 39, 577-582 (1972).

${ }^{11}$ A. H. Nayfeh, "Nonlinear Transverse Vibrations of Beams with Properties that Vary Along the Length," J. Acoust. Soc. Am. 53, 766-770 (1973).

${ }^{12} \mathrm{~S}$. Atluri, "Nonlinear Vibrations of a Hinged Beam Including Nonlinear Inertia Effects," J. Appl. Mech. 40, 121-126 (1973)

${ }^{13}$ A. H. Nayfeh, Perturbation Methods (Wiley-Interscience, New York, 1973).

${ }^{14}$ M. J. Jacobsen and R. C. W. van der Heyde, "Acoustic Fatigue Design Information for Honeycomb Panels with FiberReinforced Facings," J. Aircraft 9, No. 4, 31-42 (1972). 